\title{
Compósitos polímero-madeira preparados por polimerização in situ de metil metacrilato usando aditivos bifuncionais
}

\section{Wood-polymer composites prepared by in situ polymerization of methyl methacrylate using bi-functional additives}

\begin{abstract}
Bruno Dufau Mattos ${ }^{\text {* }}$, André Luiz Missio², Pedro Henrique Gonzalez de Cademartori Darci Alberto Gatto ${ }^{2,4}$ e Washington Luiz Esteves Magalhães ${ }^{1,5}$
\end{abstract}

${ }^{1}$ Programa Integrado de Pós-Graduação em Engenharia e Ciência dos Materiais, Universidade Federal do Paraná - UFPR, Curitiba, PR, Brasil

${ }^{2}$ Centro de Ciências Rurais, Universidade Federal de Santa Maria - UFSM, Santa Maria, RS, Brasil

${ }^{3}$ Departamento de Ciências Florestais, Universidade Federal do Paraná - UFPR, Curitiba, PR, Brasil

${ }^{4}$ Centro de Engenharias, Universidade Federal de Pelotas - UFPel, Pelotas, RS, Brasil

${ }^{5}$ Laboratório de Tecnologia da Madeira, Embrapa Florestas, Colombo, PR, Brasil

*brunodufaumattos@gmail.com

\begin{abstract}
Resumo
O presente trabalho teve por objetivo a confecção de compósitos polímero-madeira por meio de polimerização in situ de metil metacrilato (MMA), utilizando ácido metacrílico (MAA) e glicidil metacrilato (GMA) como agentes de ligação e reticulação. Amostras de madeira de guapuruvu foram impregnadas em um sistema de vácuo e pressão e polimerizadas em estufa a $90^{\circ} \mathrm{C}$ por $10 \mathrm{~h}$, usando $1,5 \%$ de peroxido de benzoíla como catalisador. Os compósitos foram caracterizados por meio de testes de absorção de água e estabilidade dimensional, molhabilidade, ATR-IR, TGA, MEV e WPG. Os espectros de ATR-IR mostraram incrementos nas bandas a 1746, 1460, e $1145 \mathrm{~cm}^{-1}$, referentes as estruturas químicas dos polímeros dentro da madeira, confirmado posteriormente pelas imagens de MEV. A termogravimetria apontou reações químicas entre os copolímeros e a parede celular da madeira nos compósitos com GMA e MAA. Os compósitos preparados com MMA apresentaram incrementos acima de 50\% nas propriedades higroscópicas e de estabilidade dimensional, entretanto a adição de GMA e MAA resultou em maiores incrementos nas mesmas propriedades, entre 66-90\%.
\end{abstract}

Palavras-chave: polimerização por radicais livres, Schizolobium parahyba, catálise térmica, reticulantes, agentes de ligação.

\begin{abstract}
We prepared wood/polymer composites by in situ polymerization of methyl methacrylate (MMA) using glycidyl methacrylate (GMA) and methacrylic acid (MAA) as cross-linkers and coupling agents. The guapuruvu wood samples were impregnated in a vacuum/pressure system and polymerized at $90{ }^{\circ} \mathrm{C}$ for $10 \mathrm{~h}$, using benzoyl peroxide at $1.5 \mathrm{wt} \%$ as catalyst. We characterized the composites through water uptake, dimensional stability tests, wettability, ATR-FTIR spectroscopy, TGA, SEM, and WPG. ATR-FTIR spectra showed an increase of the peaks at 1746,1460 , and $1145 \mathrm{~cm}^{-1}$, corresponding to the chemical structures of the polymers into the pores and capillaries of the wood. This was further confirmed in the SEM images. TGA curves showed evidences for a chemical interaction between the copolymer and the wood cell wall in the composites with GMA and MAA. Hygroscopicity and dimensional stability properties of the composites prepared with MMA improved by $50 \%$. Nevertheless, the addition of GMA and MAA resulted in additional increases for hydrophobicity and dimensional stability of the samples, $\sim 66-90 \%$.
\end{abstract}

Keywords: free-radical polymerization, Schizolobium parahyba, heat catalyst, cross-linkers, coupling agents. 


\section{Introdução}

Os problemas relacionados a higroscopicidade e instabilidade dimensional da madeira como matéria-prima podem ser suprimidos pelo desenvolvimento de compósitos polímero-madeira - podendo ser qualquer combinação entre polímero e madeira. Estes materiais podem ser de matriz polimérica (por exemplo, polipropileno, polietileno, cloreto de vinila) reforçada ou preenchida com partículas ou fibras de madeira ${ }^{[1]}$, ou podem ser madeira sólida preenchida por um polímero sintetizado in situ a partir de monômeros insaturados impregnados nos poros da madeira ${ }^{[2-6]}$.

O preparo de compósitos por polimerização in situ é promissor, no entanto, pouco difundido industrialmente em comparação aos compósitos particulados. $\mathrm{O}$ tratamento baseia-se na impregnação dos poros e capilares da madeira (ou a própria parede celular) com algum componente que formará um material polimerizado depositado nestes lugares. Para que ocorra a penetração, as substâncias impregnantes devem ter baixo peso molecular e baixa viscosidade ${ }^{[7,8]}$.

$\mathrm{O}$ interesse da utilização de metil metacrilato (MMA) na confecção destes compósitos dá-se devido ao seu baixo custo, fácil síntese, estabilidade química, translucidez, e pela facilidade de catálise. Entretanto, a falta de afinidade entre o MMA e a madeira é conhecida ${ }^{[5,9]}$, e neste caso agentes de ligação podem ser utilizados. A aplicação de ácido metacrílico (MAA) como agente reticulante de cadeia polimérica e de ligação ainda não foi testada com o objetivo de promover um copolímero reticulado e grafitado na parede celular da madeira. Por outo lado, o glicidil metacrilato (GMA) já foi utilizado para este propósito e mostrou bons resultados ${ }^{[10-12]}$. A inserção de novos aditivos em compósitos polímero-madeira deve ser estudada, entretanto um comparativo com aditivos já conhecidos é interessante para avaliar a aplicabilidade das novas formulações.

Quanto a madeira, o requisito para a seleção é a sua tratabilidade, ou seja, a capacidade de ser impregnada estimativas quanto a tratabilidade são feitas conhecendo a massa específica, porosidade, permeabilidade e estrutura anatômica da espécie. Para este estudo utilizou-se o guapuruvu (Schizolobium parahyba (Vell.) Blake) devido à alta tratabilidade da madeira e da grande produtividade da espécie em plantios comerciais, próximo de $45 \mathrm{~m}^{3} / \mathrm{ha}^{\text {ano }}{ }^{[13]}$.

Nesse contexto, o presente trabalho teve por objetivo avaliar as propriedades termoquímicas e higroscópicas de compósitos polímero-madeira preparados por polimerização in situ de metil metacrilato (MMA) na madeira de Guapuruvu, utilizando ácido metacrílico (MAA) e glicidil metacrilato (GMA) como agentes de ligação e reticulação.

\section{Material e Métodos}

\subsection{Material}

A madeira de guapuruvu foi selecionada de plantios experimentais homogêneos evitando a seleção de árvores de bordadura, tortuosas e bifurcadas. Três árvores foram desdobradas para a obtenção de pranchas centrais de $8 \times 50 \times 200 \mathrm{~cm}^{3}$ (espessura, largura e comprimento), com medula inclusa ao centro e bem orientada no sentido longitudinal. Deste material, foram confeccionados 150 corpos de prova livres de defeitos com dimensões de $1,5 \times 1,5 \times 2,5 \mathrm{~cm}^{3}$ (espessura, largura e comprimento). Em cada tratamento utilizou-se 40 corpos de prova: 15 para absorção de água e estabilidade dimensional, 15 para ganho percentual de massa, 5 para molhabilidade e 5 para os ensaios qualitativos.

Os monômeros utilizados foram o metil metacrilato (MMA), SA\# M55909 (99\%, <30ppm de MEHQ como inibidor), glicidil metacrilato (GMA), SA\# 151238 (97\%, $100 \mathrm{ppm}$ de MEHQ como inibidor) e o ácido metacrílico (MAA), SA\# 155721 (99\%, 250 ppm de MEHQ como inibidor) obtidos na Sigma-Aldrich. Como catalisador, utilizou-se o peróxido de benzoíla (PBO) obtido na Vetec Química Fina LTDA.

\subsection{Preparação dos compósitos}

Antes da impregnação as amostras foram secas a $90 \pm 2{ }^{\circ} \mathrm{C}$ até massa constante ( 2 dias) e as soluções foram preparadas de acordo com as proporções apresentadas na Tabela 1. A massa de catalisador foi misturada utilizando agitação magnética simples.

Para a impregnação, as amostras secas foram inseridas em um cilindro de tratamento e submetidas a um vácuo inicial de $600 \mathrm{mmHg}$ por $30 \mathrm{~min}$ com o objetivo de retirar o máximo de ar dos poros e capilares da madeira. Ainda sob vácuo, a solução foi inserida no cilindro por diferença de pressão e em seguida o sistema foi pressurizado a $6 \mathrm{~atm}$ por 1h, utilizando-se um compressor de ar. Por fim, a solução foi escoada para fora do cilindro e a madeira impregnada foi retirada do cilindro. $\mathrm{O}$ excesso de solução foi deixado escorrer por $10 \mathrm{~min}$ e a polimerização foi realizada em estufa a $90 \pm 2^{\circ} \mathrm{C}$ por $10 \mathrm{~h}$

De acordo com Li et al. ${ }^{[12]}$ e Zhang et al. ${ }^{[14]}$ as ligações químicas esperadas entre a madeira e os copolímeros de poli(MMA-co-GMA) e poli(MMA-co-MAA), ocorrem respectivamente, via abertura do anel epóxi terminal (GMA) e grupo carboxílico (MAA) com as hidroxilas livres da madeira.

\subsection{Ganho percentual de massa}

O ganho percentual de massa dos compósitos foi calculado de acordo com a Equação 1, como previamente mencionado em outros estudos ${ }^{[5,6,15,16]}$.

$$
\mathrm{CP}_{\%}=\left[\frac{\left(\mathrm{m}_{\mathrm{p}}-\mathrm{m}_{0}\right)}{\mathrm{m}_{0}}\right] * 100
$$

Em que: $\mathrm{m}_{\mathrm{p}}=$ massa de amostra após o tratamento; $\mathrm{m}_{0}=$ massa da amostra antes do tratamento.

Tabela 1. Especificação dos compósitos polímero-madeira.

\begin{tabular}{|c|c|c|c|c|}
\hline \multirow{2}{*}{$\begin{array}{c}\text { Código do } \\
\text { Tratamento }\end{array}$} & \multicolumn{3}{|c|}{$\%$} & \multirow{2}{*}{$\begin{array}{c}\text { massa } \% \\
\text { PBO }\end{array}$} \\
\hline & МMA & GMA & МАA & \\
\hline MMA & 100 & - & - & 1,5 \\
\hline M:G (2:1) & 66,5 & 33,5 & - & 1,5 \\
\hline M:G (3:1) & 75 & 25 & - & 1,5 \\
\hline $\mathrm{M}: \mathrm{A}(2: 1)$ & 66,5 & - & 33,5 & 1,5 \\
\hline $\mathrm{M}: \mathrm{A}(3: 1)$ & 75 & - & 25 & 1,5 \\
\hline
\end{tabular}

MMA $=$ metil metacrilato; $\mathrm{GMA}=$ glicidil metacrilato; $\mathrm{MAA}=$ ácido metacrílico; $\mathrm{PBO}=$ peróxido de benzoíla. 


\subsection{Morfologia por microscopia eletrônica de varredura}

A morfologia dos compósitos foi avaliada por meio de um microscópico eletrônico de varredura (MEV) Shimadzu modelo SSX 550 Superscan. A superfície transversal das amostras foi preparada com um micrótomo de deslizamento Leica modelo SM2010R e logo em seguida foram secas em estufa a $103 \pm 2^{\circ} \mathrm{C}$ até massa constante. Antes da obtenção das imagens, a superfície das amostras foi metalizada por meio da deposição de pó de ouro.

\subsection{Espectroscopia no infravermelho (ATR-IR)}

Utilizou-se um equipamento Nicolet Nexus 570, equipado com um instrumento MKII Golden Gate SPECAC, com o dispositivo de refletância total atenuada (ATR) fixado na parte superior à uma unidade de condensação do feixe ótico com cristal de ZnSe. Os espectros de ATR-IR foram obtidos de amostras moídas ( $<60$ mesh), utilizando resolução de $4 \mathrm{~cm}^{-1}, 32$ varreduras, ângulo de incidência fixo em $75^{\circ} \mathrm{e}$ amplitude de leitura de 700 a $4000 \mathrm{~cm}^{-1}$.

O alinhamento da lâmpada e o background foram coletados antes de todos os testes e os espectros apresentados foram normalizados pelo máximo. A apresentação dos espectros foi feita pela média de três mensurações para cada tratamento.

\subsection{Termogravimetria}

As curvas termogravimétricas (TG) e as respectivas derivadas (DTG) foram obtidas em um equipamento DTG-60 da Shimadzu. Na condução dos ensaios utilizou-se atmosfera inerte de nitrogênio (fluxo de gás de $50 \mathrm{ml} \cdot \mathrm{min}^{-1}$ ), amplitude de temperatura de 25 a $600^{\circ} \mathrm{C}$ e taxa de aquecimento de $10^{\circ} \mathrm{C}$. $\mathrm{min}^{-1}$. Utilizou-se entre 5 e $8 \mathrm{mg}$ de amostra e recipiente de platina para alocação da amostra dentro do equipamento.

\subsection{Absorção de água e estabilidade dimensional}

A hidrofobicidade e a estabilidade dimensional do material foi avaliada pela eficiência de repelência à água (WRE) e pela eficiência anti-inchamento (ASE). Inicialmente, as amostras foram secas em estufa a $103 \pm 2^{\circ} \mathrm{C}$ (até massa constante) para padronizar a condição de umidade inicial.

Os níveis de eficiência de repelência à água (WRE) e de anti-inchamento (ASE) dos compósitos foram obtidos durante quatro ciclos de imersão-secagem. Em cada ciclo, a massa e o volume das amostras foram mensurados no tempo zero (amostras secas a $103 \pm 2^{\circ} \mathrm{C}$ ) e após $96 \mathrm{~h}$ de imersão. Com os dados obtidos, a WRE e a ASE foram calculadas pelas Equações 2 e 3, respectivamente.

$$
\mathrm{WRE}=\left[\frac{\left(\Delta \mathrm{m}_{\mathrm{nt}}-\Delta \mathrm{m}_{\mathrm{t}}\right)}{\Delta \mathrm{m}_{\mathrm{nt}}}\right] * 100
$$

onde: $\Delta \mathrm{m}=\left(\mathrm{m}_{\mathrm{u}}-\mathrm{m}_{\mathrm{s}}\right) / \mathrm{m}_{\mathrm{s}}$

$$
\operatorname{ASE}=\left[\frac{\left(\Delta \mathrm{V}_{\mathrm{nt}}-\Delta \mathrm{V}_{\mathrm{t}}\right)}{\Delta \mathrm{V}_{\mathrm{nt}}}\right] * 100
$$

onde: $\Delta \mathrm{V}=\left(\mathrm{V}_{\mathrm{u}}-\mathrm{V}_{\mathrm{s}}\right) / \mathrm{V}_{\mathrm{s}}$

onde: $\Delta \mathrm{V}=$ variação de volume; $\mathrm{V}_{\mathrm{u}}=$ volume da amostra úmida $\left(\mathrm{cm}^{3}\right) ; \mathrm{V}_{\mathrm{s}}=$ volume da amostra seca $\left(\mathrm{cm}^{3}\right) ; \mathrm{t}=$ tratada (compósito); $\mathrm{nt}=$ não tratada; $\Delta \mathrm{m}=$ variação da massa; $\mathrm{m}_{\mathrm{u}}=$ massa da amostra úmida $(\mathrm{g}) ; \mathrm{m}_{\mathrm{s}}=$ massa da amostra seca $(\mathrm{g})$.

Após cada ciclo de imersão-secagem, calculou-se a lixiviação $\left(\mathrm{L}_{\%}\right)$ de produtos solúveis em água e material não polimerizado presentes nas amostras.

\subsection{Molhabilidade}

A molhabilidade da superfície das amostras foi avaliada pela técnica de ângulo de contato, por meio de um goniômetro Dataphysics modelo 0CA configurado para a aplicação do método da gota séssil. Os ensaios foram conduzidos pela deposição de gotículas de $5 \mu \mathrm{lem}$ três pontos distintos, nos planos anatômicos longitudinal radial e tangencial, totalizando 15 mensurações por tratamento. Para construir uma cinética de molhabilidade, as mensurações foram realizadas após 5 , $20,35,50,65,80$ e 95 segundos de contato entre gotícula e a superfície da amostra.

\subsection{Análise estatística}

Primeiramente foram verificadas a normalidade, homogeneidade de variância dos dados e independência dos erros pelos testes White, Shapiro-Wilk e Durbin-Watson, respectivamente. A partir dos resultados, verificou-se que todos as análises atenderam os pressupostos para realização dos testes paramétricos. Dessa forma, os dados foram interpretados através de análise de variância (ANOVA), em que foram realizadas comparações de médias, entre os seis tratamentos, pelo teste $\mathrm{F}$ em $5 \%$ de significância. Em caso de rejeição da hipótese nula, foi realizado teste de médias HSD (Honestly Significant Difference) de Tukey, em 5\% de significância.

\section{Resultados e Discussão}

\subsection{Morfologia dos compósitos}

O ganho percentual de massa, após o tratamento, observado para o compósito MMA foi estatisticamente menor que o dos demais, exceto em relação ao compósito M:A 2:1 (Tabela 2). Essa diferença pode ser atribuída a falta de afinidade entre o poli(MMA) e a madeira.

Os compósitos com GMA e MAA na composição apresentaram ganho percentual de massa significativamente mais elevado devido à maior compatibilidade entre os copolímeros e a madeira, o que permitiu a penetração do reforço polimérico na parede celular da madeira. $\mathrm{O}$ aumento do ganho percentual de massa após a inserção de agentes de ligação foi previamente reportado por outros autores ${ }^{[12,16,17]}$.

O elevado ganho percentual de massa ( $>100 \%)$, observado para todos compósitos, pode resultar numa diminuição do índice de mérito deste material (razão entre resistência mecânica e densidade), uma vez que, após tratamentos de polimerização in situ, comumente observa-se incrementos nas propriedades mecânicas abaixo de $100 \%{ }^{[5]}$.

O efeito da polimerização in situ na microestrutura da madeira de guapuruvu, ocorreu de diferentes formas entre os compósitos (Figura 1). Originalmente, a madeira de guapuruvu apresenta vasos como os principais elementos anatômicos, os quais são os principais responsáveis pelo fluxo de líquidos em folhosas ${ }^{[18]}$. 
Tabela 2. Ganho percentual de massa após os tratamentos.

\begin{tabular}{ccccccccc}
\hline & \multicolumn{9}{c}{ Compósitos } & \multirow{2}{*}{ Razão F } & \multirow{2}{*}{ Valor p } \\
\cline { 2 - 5 } & MMA & M:G 2:1 & M:G 3:1 & M:A 2:1 & M:A 3:1 & & \multirow{2}{*}{0,01} \\
\hline Média (\%) & $116,1 \mathrm{a}$ & $158,8 \mathrm{c}$ & $164,1 \mathrm{c}$ & $129,5 \mathrm{ab}$ & $151,9 \mathrm{bc}$ & \multirow{2}{*}{4,79} & \\
DP & 22,0 & 22,9 & 39,5 & 28,9 & 30,8 & & \\
\hline
\end{tabular}

Valores seguidos por letras iguais não apresentam diferença estatisticamente significativa, de acordo com o teste HSD de Tukey, em nível de $5 \%$ de probabilidade de erro. $\mathrm{DP}=$ desvio padrão.

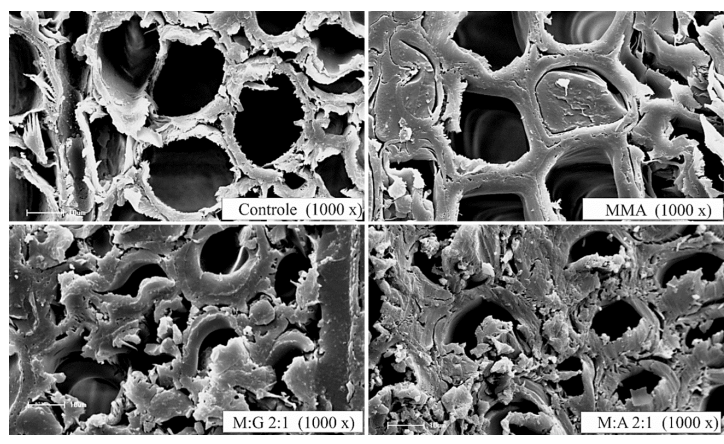

Figura 1. Morfologia da microestrutura dos compósitos confeccionados por polimerização in situ.

Para o compósito MMA notou-se espaços vazios entre a parede celular da madeira e o poli(MMA) devido à contração do polímero após a síntese e a falta de afinidade entre ambos. Islam et al. ${ }^{[19]}$ observaram imagens similares utilizando outras espécies.

Nos compósitos M:G e M:A (ambas frações) ocorreu um recobrimento polimérico na parede celular da madeira, entretanto o preenchimento dos elementos celulares permaneceu incompleto. O preenchimento parcial dos vasos e poros da madeira de guapuruvu, nos compósitos M:G e M:A, ocorreu pela penetração de moléculas polares do GMA e MAA no interior da parede celular, o que só é possível devido à reatividade entre o grupo carboxílico do MAA e o anel epóxi do GMA com os grupos $\mathrm{OH}$ da madeira. A penetração de polímeros na parede celular acarreta, normalmente, em incrementos mais significativos nas propriedades da madeira ${ }^{[11]}$.

\subsection{Avaliação termoquímica dos compósitos}

Nos espectros de ATR-IR para os polímeros (Figura 2a), foram observados picos característicos de grupos metacrilatos e metacrílicos. Observou-se um pico acentuado de forte intensidade a $1730 \mathrm{~cm}^{-1}$, correspondente as vibrações de estiramento do grupo $\mathrm{C}=\mathrm{O}$, um pico largo e intenso a $1145 \mathrm{~cm}^{-1}$, referente ao estiramento $\mathrm{C}-\mathrm{O}$ do grupo éster e um pico a $1460 \mathrm{~cm}^{-1}$ referente a deformação $\mathrm{C}-\mathrm{H}$.

Para a madeira controle, a região entre 1700 e $1750 \mathrm{~cm}^{-1}$, corresponde às carbonilas $(\mathrm{C}=\mathrm{O})$ dos grupos acetil $\mathrm{e}$ ácidos carboxílicos presentes nas hemiceluloses e lignina, respectivamente ${ }^{[20]}$. Além disso, os grupos carboxílicos estão presentes nos extrativos da madeira, principalmente nos ácidos $\operatorname{graxos}^{[21]}$. Nos espectros ATR-IR dos compósitos, observou-se incremento significativo na intensidade da banda a $1746 \mathrm{~cm}^{-1}$ devido à presença de novos grupos $\mathrm{C}=\mathrm{O}$ conjugados oriundos do reforço polimérico (Tabela 3 ).

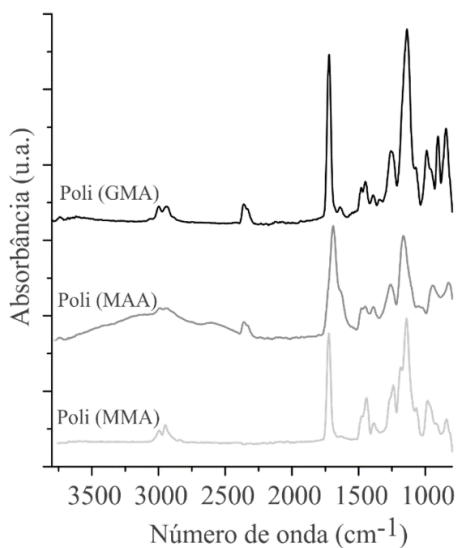

(a)

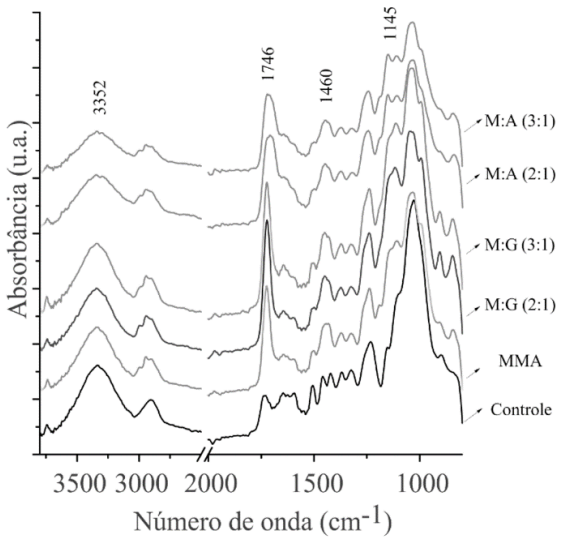

(b)

Figura 2. Espectros de ATR-IR para os polímeros (a), madeira controle e compósitos (b).

Para a construção das razões entre picos foi utilizado o pico a $1050 \mathrm{~cm}^{-1}$ como padrão interno. Este pico corresponde a deformação no plano das estruturas $\mathrm{C}-\mathrm{H}$ e estiramento simétrico C-O da celulose - duas estruturas altamente estáveis em tratamentos térmicos ou químicos ${ }^{[22,23]}$.

Por meio dos valores para a razão dos picos 1460 e $1145 \mathrm{~cm}^{-1}$ ficou comprovado o aumento das intensidades de absorção correspondentes, respectivamente, à deformação da estrutura $\mathrm{C}-\mathrm{H}$ e ao estiramento $\mathrm{C}-\mathrm{O}$ do grupo éster das estruturas poliméricas utilizadas como reforço (Tabela 3). Verificou-se maior intensidade da banda a $1145 \mathrm{~cm}^{-1}$ nos compósitos com GMA e MAA. Tal observação indica a ocorrência de ligações químicas entre copolímeros e madeira, uma vez que a ligação C-O é típica da reticulação entre os dois materiais. $\mathrm{O}$ aumento da intensidade deste pico para os compósitos 
Tabela 3. Razões para os picos correspondentes às estruturas poliméricas.

\begin{tabular}{ccccc}
\hline Amostra & $\mathbf{I}_{\mathbf{1 7 4 6 / 1 0 5 0}}$ & $\mathbf{I}_{\mathbf{1 1 4 5 / 1 0 5 0}}$ & $\mathbf{I}_{\mathbf{1 4 6 0 / 1 0 5 0}}$ & $\mathbf{I}_{\mathbf{3 3 5 2 / 1 0 5 0}}$ \\
\hline Controle & 0,215 & 0,416 & 0,293 & 0,338 \\
MMA & 0,506 & 0,728 & 0,344 & 0,351 \\
M:G 2:1 & 0,529 & 0,801 & 0,339 & 0,306 \\
M:G 3:1 & 0,521 & 0,771 & 0,359 & 0,322 \\
M:A 2:1 & 0,477 & 0,761 & 0,377 & 0,305 \\
M:A 3:1 & 0,496 & 0,809 & 0,365 & 0,318 \\
\hline
\end{tabular}

Em que: $\mathrm{I}=$ intensidade.

com GMA e MAA também foi comprovado pela razão de picos (Tabela 3).

A banda a $3352 \mathrm{~cm}^{-1}$, referente ao estiramento $\mathrm{OH}$, não apresentou modificação no compósito MMA, entretanto para os compósitos com GMA e MAA observou-se um ligeiro deslocamento desta banda para números de onda mais baixos $\left(\sim 3328 \mathrm{~cm}^{-1}\right)$. Essa pequena modificação deu-se pela conversão parcial dos grupos hidroxila em novos grupos éter e o aparecimento de novas estruturas $\mathrm{OH}$ oriundas dos copolímeros ${ }^{[1,12]}$. Nos compósitos M:A e M:G, para ambas frações, observou-se uma diminuição significativa da razão $I_{3352 / 1050}$, o que indicou substituição parcial dos grupos $\mathrm{OH}$ da madeira durante a reticulação oriunda da reação de copolimerização.

Não foram observadas alterações significativas nos picos a $1321,1230,1157,896 \mathrm{~cm}^{-1}$ referente aos polissacarídeos da madeira, e nos picos a 1592 e $1504 \mathrm{~cm}^{-1}$, referentes às estruturas de lignina. A ausência de modificações nestes picos, após a polimerização in situ, contrasta com o observado em outros tratamentos também utilizados com o objetivo de incrementar a hidro repelência da madeira ${ }^{[24,25]}$.

Em continuação à análise termoquímica fez-se uma avaliação comparativa do desempenho térmico da madeira controle com os compósitos (Figura 3b). Para isso, foram adicionados termogramas referentes ao poli(MMA), poli(GMA) e poli(MAA) à discussão (Figura 3a).

Os picos a 288 e $371^{\circ} \mathrm{C}$ (DTG 1 e 2) correspondem aos dois principais estágios de decomposição térmica do poli(MMA). O pico 1 é comum entre o poli(MMA) e poli(GMA), e refere-se à decomposição térmica do grupo metacrilato, enquanto que as ramificações metil e glicidil decompõe-se em temperaturas diferentes. A segunda decomposição térmica do poli(GMA) foi observada a $421^{\circ} \mathrm{C}$ (DTG 3). Para o poli(MAA) foi observado um único pico intenso a $428^{\circ} \mathrm{C}$ (DTG 4).

Em geral, os polímeros apresentaram baixa estabilidade térmica $\left(\mathrm{T}_{\mathrm{i}}\right.$, Tabela 4$)$, como previamente reportado por Ferriol et al. ${ }^{[26]}$, von Lampe et al. ${ }^{[27]}$ e Zulfiqar et al. ${ }^{[28]}$. Entretanto, é importante ressaltar que o desempenho térmico dos copolímeros de MMA, GMA e MAA pode ser ligeiramente diferente. Por exemplo, de acordo com Vinu e Madras ${ }^{[29]}$, o poli (MMA-co-MAA) apresenta um único estágio de decomposição a $400^{\circ} \mathrm{C}$. Na pirólise de polímeros, o principal fenômeno que ocorre é a despolimerização da cadeia com a formação de estruturas como álcoois e anidridos, entretanto na decomposição do poli(MMA-co-MAA) existe um mecanismo diferente. Neste caso especifico, ocorre uma

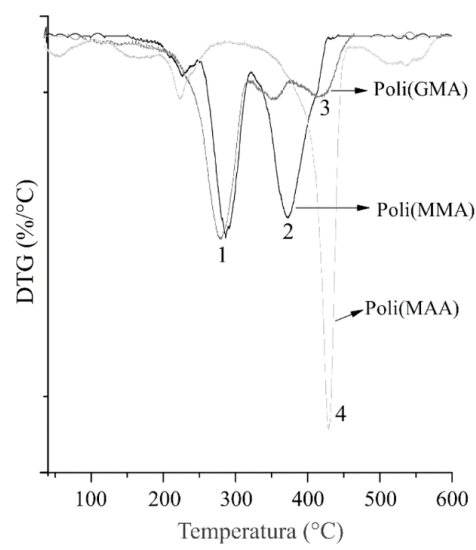

(a)

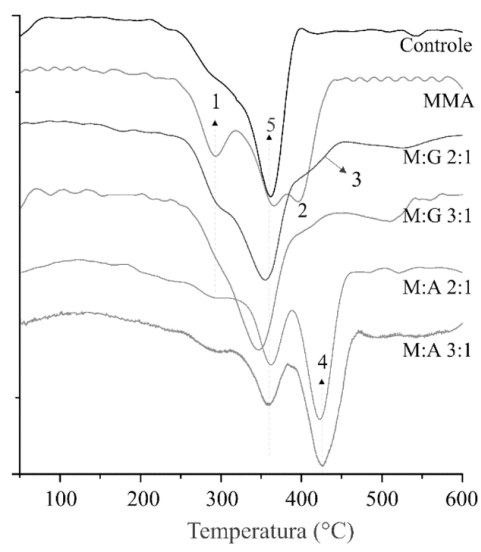

(b)

Figura 3. Curvas de DTG para os polímeros (a), madeira controle e compósitos (b).

ciclização intramolecular entre o éster adjacente do MMA e o grupo carboxílico do MAA, resultando na formação de uma estrutura anidrido em forma de anel, que necessita de maior energia térmica para a decomposição.

Por outro lado, o poli(MMA-co-GMA) apresenta desempenho térmico intermediário ao poli(MMA) e poli(GMA). Dois picos de degradação são observados, um para cada componente, e conforme a diminuição da proporção de GMA o copolímero tende a ser mais estável termicamente ${ }^{[28]}$.

Nos termogramas dos compósitos são observados caraterísticas do comportamento térmico dos polímeros e da madeira sem tratamento. Verificou-se que os compósitos apresentaram $\mathrm{T}_{\mathrm{i}}$ inferior às madeiras controles, conforme anteriormente verificado por $\mathrm{Li}$ et al. ${ }^{[30]}$, o que ocorre devido à baixa estabilidade térmica dos polímeros. Ainda nesse sentido, pode-se afirmar que a presença dos agentes de ligação em menor carga (3:1) acarretou em $\mathrm{T}_{\mathrm{i}}$ mais precoces.

Tal fato vai de encontro com o observado por Devi et al. ${ }^{[3]]}$ e Devi e Maji ${ }^{[32]}$, ao utilizarem o GMA como aditivo no tratamento da madeira com estireno. Os autores observaram um ligeiro aumento na $\mathrm{T}_{\text {i }}$, alterando de $277^{\circ} \mathrm{C}$ da madeira não tratada para 280,283 e $288^{\circ} \mathrm{C}$ para os compósitos 
Tabela 4. Resumo da análise termogravimétrica.

\begin{tabular}{|c|c|c|c|c|c|c|c|c|c|c|c|}
\hline \multirow[t]{2}{*}{ Amostras } & \multirow[t]{2}{*}{$\mathbf{T i}$} & \multicolumn{5}{|c|}{ Picos DTG $\left({ }^{\circ} \mathrm{C}\right)$} & \multicolumn{4}{|c|}{$\begin{array}{c}\text { Temperatura para } X \% \text { de perda de } \\
\text { massa }\left(T_{d}\right)\end{array}$} & \multirow{2}{*}{$\begin{array}{l}\text { MR } \\
(\%)\end{array}$} \\
\hline & & 1 & 2 & 3 & 4 & 5 & 40 & 50 & 60 & 70 & \\
\hline Poli(MMA) & 215 & 288 & 373 & - & - & - & 303 & 339 & 360 & 372 & 4,7 \\
\hline Poli(GMA) & 142 & 279 & - & 421 & - & - & 285 & 297 & 328 & 363 & 9,7 \\
\hline Poli(MAA) & 53 & - & - & - & 429 & - & 404 & 418 & 425 & 430 & 2,2 \\
\hline Controle & 275 & - & - & - & - & 360 & 351 & 360 & 369 & 384 & 20,4 \\
\hline MMA & 270 & 294 & 397 & - & - & 365 & 363 & 377 & 393 & 408 & 19,4 \\
\hline M:G 2:1 & 267 & 291 & - & 411 & - & 355 & 341 & 352 & 364 & 387 & 9,1 \\
\hline M:G 3:1 & 260 & 290 & - & 411 & - & 345 & 347 & 359 & 373 & 402 & 10,2 \\
\hline M:A 2:1 & 264 & 290 & - & - & 423 & 361 & 383 & 411 & 425 & 437 & 6,2 \\
\hline M:A 3:1 & 222 & 290 & - & - & 425 & 357 & 406 & 421 & 433 & 455 & 7,3 \\
\hline
\end{tabular}

$\mathrm{Ti}=$ temperatura de inicio da decomposição $(3 \%$ de perda $) ; \mathrm{Td}=$ temperatura de decomposição; $\mathrm{MR}=$ massa residual a $600^{\circ} \mathrm{C}$.

com estireno, estireno-GMA (2:1) e estireno-GMA (1:1), respectivamente. Alguns fatores, como a alta estabilidade térmica do polímero de estireno (devido sua estrutura aromática) e a baixa carga de polímeros nos compósitos ( $25 \%$ ) podem ter influenciado esse resultado.

Na curva DTG para o compósito MMA notou-se a presença de três picos distintos (picos DTG 1, 2 e 5). Os dois primeiros foram relacionados às etapas de decomposição térmica do poli(MMA), enquanto que o terceiro corresponde à decomposição térmica das fibras de madeira. A presença destes três picos isolados na curva de decomposição térmica do compósito, indicou ausência de ligação química entre o poli(MMA) e a madeira.

Já para os compósitos M:G (em ambas frações) foi observado desempenho térmico similar ao controle, indicando que o copolímero se decompôs junto da madeira e, além disso, houve um deslocamento da temperatura de decomposição térmica das fibras para temperaturas mais amenas. A alteração da temperatura de decomposição das fibras indica uma degradação simultânea do polímero e madeira, como um novo material.

Nos compósitos M:A, devido à baixa estabilidade térmica do poli(MAA) e pela baixa energia de ligação entre o $\mathrm{COOH}$ e $\mathrm{OH}$, os efeitos da inserção do MAA como aditivo acoplante só aparecem em estágios mais avançados da decomposição térmica, acima de $40 \%$ de perda de massa, conforme observado pela $\mathrm{T}_{\mathrm{d}}$ (Tabela 4). Isso ocorreu mesmo considerando uma baixa $\mathrm{T}_{\mathrm{i}}$ para estes compósitos. Com isso, é correto afirmar que o MAA promoveu maior interação entre o polímero e a superfície interna da madeira, entretanto de forma menos significativa que o GMA. Ainda nesse sentido, os compósitos M:A não apresentaram o primeiro pico de degradação, referente ao MMA, sugerindo que o MAA foi eficiente na reticulação da cadeia polimérica.

A massa residual a $600^{\circ} \mathrm{C}(\mathrm{MR})$ dos compósitos foi intermediária à dos polímeros e madeira controle, como previamente reportado por Devi e Maji ${ }^{[32]}$, Devi et al. ${ }^{[31]} \mathrm{e}$ Li et al. ${ }^{[30]}$. Entre os compósitos, a MR diminuiu com o aumento da fração de GMA ou MAA, corroborando que a ligação química entre copolímeros (menos estáveis termicamente) e madeira acarreta em uma degradação térmica simultânea, considerando a baixa MR dos polímeros.

\subsection{Higroscopicidade, hidrofobicidade e estabilidade dimensional}

De maneira geral, os planos tangencial e radial apresentaram comportamento muito similar para o ângulo de contato, sendo tal fator sobreposto pelo efeito dos polímeros e copolímeros na superfície dos compósitos. Para todas as curvas de molhabilidade, os valores foram máximos aos $5 \mathrm{~s}$ e decaíram até $95 \mathrm{~s}$, igualmente ao reportado por outros autores ${ }^{[33,34]}$.

Para a madeira controle, ao início do ensaio a média do ângulo de contato foi de $\sim 45^{\circ}$ e ao final o ângulo diminuiu para próximo de $0^{\circ}$. Entretanto, no compósito MMA o ângulo de contato inicial foi de $\sim 90^{\circ}$, com uma ligeira diminuição ao final do ensaio, permanecendo entre $80-85^{\circ}$ (Figura 4). Resultados semelhantes a estes foram observados por Mamiński et al. ${ }^{[35]}$ em compósitos confeccionados pelo tratamento da madeira de Beech (Fagus sylvatica L.) com butil metacrilato e butil acrilato, e também por Li et al. ${ }^{[30]}$ para compósitos de Poplar (Populuss ussuriensis Korn) confeccionados via polimerização in situ de poli(MMAco-estireno). Evidenciou-se que mesmo com polímeros sem afinidade química com a madeira, é possível aumentar significativamente a hidrofobicidade superficial da madeira.

Com a inserção de GMA como reticulante e ligante, o ângulo de contato aumentou ligeiramente para ambas frações, M:G 2:1 e M:G 3:1. Verificou-se que ao início do teste o ângulo foi de $90-95^{\circ}$ e aos $95 \mathrm{~s}$ de deposição caiu para $85-90^{\circ}$. No entanto, a copolimerização in situ de poli(MMA-co-MAA), com frações de 2:1 e 3:1, apresentaram resultados menos satisfatórios para o ângulo de contato quando comparados aos demais compósitos, mostrando maior molhabilidade.

Foi constatado que ao final da cinética de molhabilidade para a madeira controle o volume da gotícula ficou abaixo de $10 \%$, o que evidencia que além do espalhamento houve um forte fenômeno de absorção da gotícula pela madeira. Para os compósitos, a permanência do volume da gotícula próximo de $100 \%$, durante todo teste, evidenciou que a pequena diminuição do ângulo de contato para estas amostras deu-se por espalhamento superficial da gotícula, sendo ausente o fenômeno de absorção. Dito de outro modo, pode-se afirmar que os níveis de repelência à água na superfície dos compósitos são extensivamente superiores aos controles. 
Além do incremento na hidrofobicidade superficial (Figura 4), os compósitos propostos foram aptos a diminuir drasticamente a absorção de água da madeira, conforme observado nos resultados para WRE e ASE ao final dos ciclos de imersão e secagem (Tabela 5). Os polímeros formados no interior das amostras formaram uma barreira contra umidade, entretanto, como ainda ocorre absorção de água, constata-se que a polimerização in situ não elimina totalmente a higroscopicidade.

Os compósitos com GMA e MAA apresentaram resultados estatisticamente superiores para o $\mathrm{WRE}_{4^{\circ}}$ e o $\mathrm{ASE}_{4^{\circ}}$, quando comparado ao tratamento com MMA, à exceção do compósito M:A 2:1. Estes resultados mostraram que a inserção de MAA para a reticulação e ligação de MMA deve ser em baixas proporções, uma vez que o polímero resultante é altamente hidrofílico.

Além disso, os resultados de WRE e ASE ao longo dos quatro ciclos de imersão e secagem (Figura 5), evidenciam que os compósitos M:G 2:1 e M:G 3:1 são os mais eficientes quanto a exclusão de água e para aumentar a estabilidade dimensional, mantendo seus valores constantes durante os quatro ciclos. Já o compósito MMA diminuiu gradualmente os valores para WRE e ASE, enquanto que a massa de lixiviado aumentou (Figura 5).

A falta de ligações químicas entre o poli(MMA) e a madeira acarretou em lixiviação excessiva de polímeros, o que consequentemente diminuiu os resultados de WRE e ASE para o compósito MMA. Novamente o compósito M:A 2:1 mostrou resultados similares ao compósito MMA.

$\mathrm{O}$ efeito positivo dos agentes de ligação sob WRE e ASE deu-se devido a maior afinidade do reforço polimérico com a madeira o que possibilidade um recobrimento interno da parede celular. Este recobrimento age principalmente impedindo a entrada de água e diminuindo o contato da água com a madeira, o que acarreta também na diminuição do inchamento das amostras ${ }^{[8]}$.
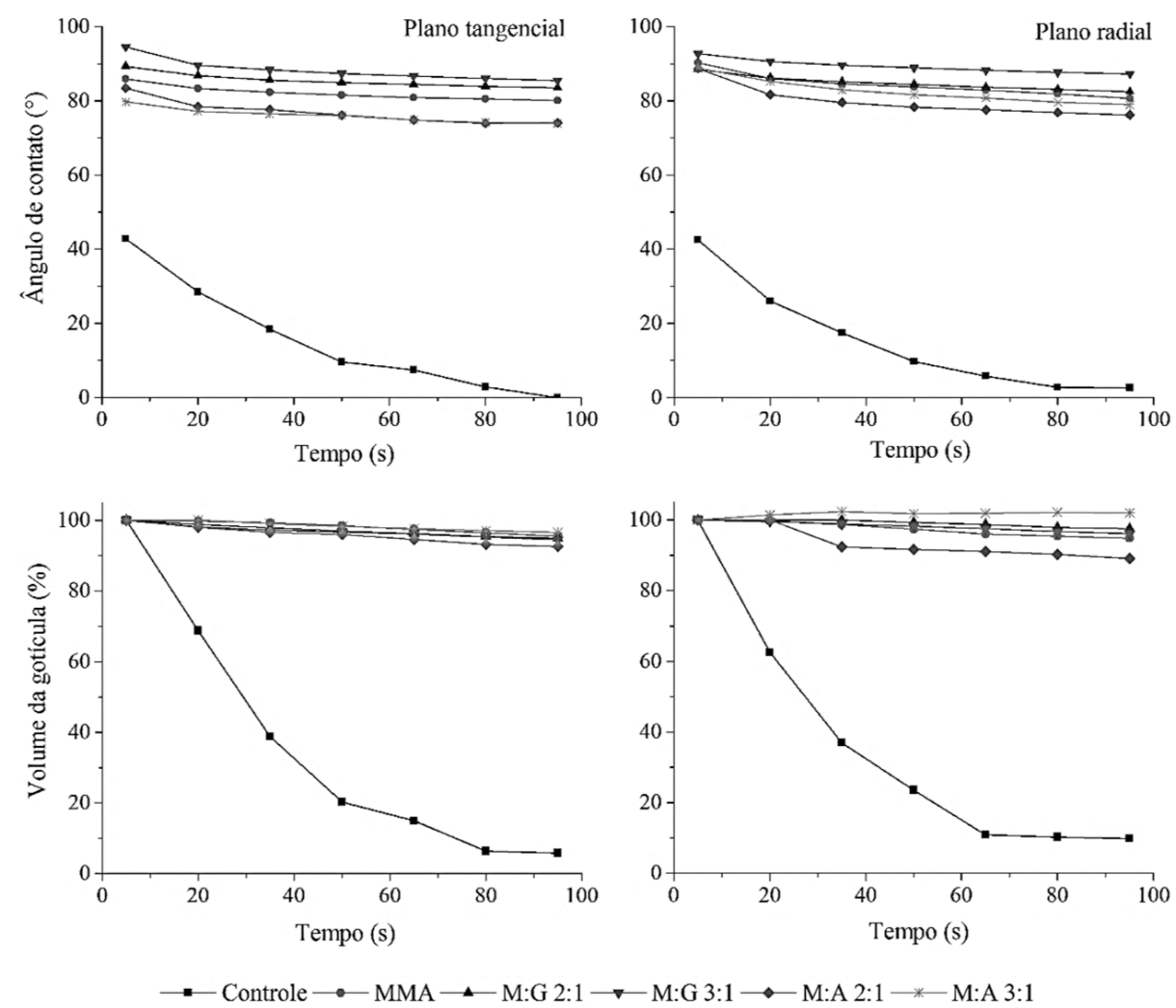

Figura 4. Molhabilidade dos compósitos e madeira controle.

Tabela 5. Parâmetros de absorção de água e estabilidade dimensional dos compósitos após o quarto ciclo de imersão e secagem.

\begin{tabular}{|c|c|c|c|c|c|c|c|}
\hline \multirow{2}{*}{ Propriedade } & & \multicolumn{5}{|c|}{ Compósito } & \multirow{2}{*}{ Razão F* } \\
\hline & & MMA & M:G 2:1 & M:G 3:1 & M: A 2:1 & M: A 3:1 & \\
\hline \multirow{2}{*}{$\mathrm{WRE}_{4^{\circ}}$} & Média & $79,2 \mathrm{a}$ & $89,8 \mathrm{~b}$ & $89,0 \mathrm{~b}$ & $81,3 \mathrm{a}$ & $90,0 \mathrm{~b}$ & \multirow{2}{*}{13,51} \\
\hline & DP & 6,3 & 2,3 & 2,5 & 6,7 & 1,6 & \\
\hline \multirow{2}{*}{$\operatorname{ASE}_{4^{\circ}}$} & Média & $62,8 \mathrm{a}$ & $79,3 \mathrm{~b}$ & $80,7 \mathrm{~b}$ & $66,7 \mathrm{a}$ & $72,5 \mathrm{ab}$ & \multirow{2}{*}{4,60} \\
\hline & DP & 7,9 & 16,5 & 9,4 & 9,8 & 11,5 & \\
\hline
\end{tabular}

*Valores na coluna seguidos por letras iguais não apresentam diferença estatisticamente significativa, de acordo com o teste HSD de Tukey, em nível de $1 \%$ de probabilidade de erro. $4^{\circ}=$ quarto ciclo de imersão e secagem. 


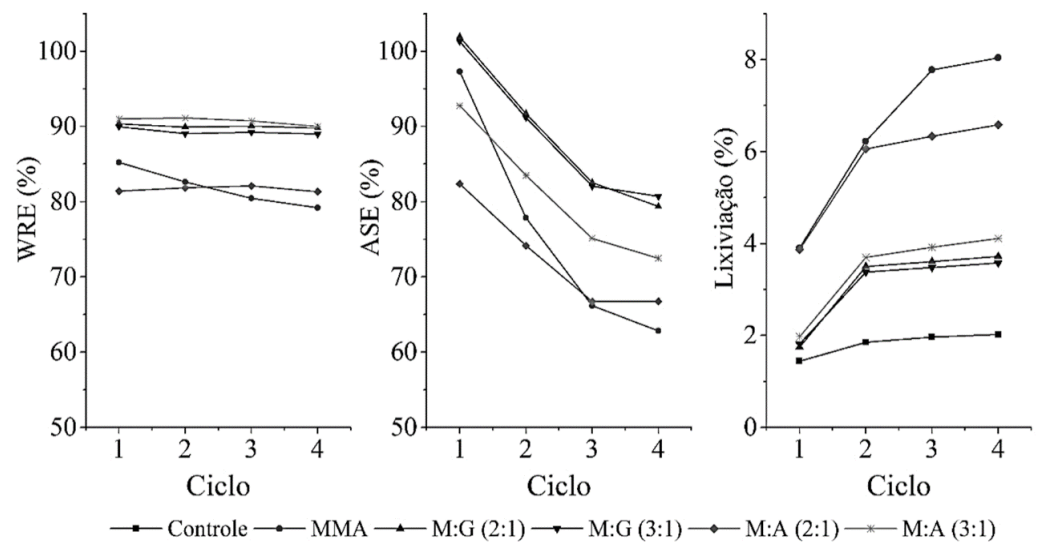

Figura 5. Eficiência de repelência à agua (WRE), anti-inchamento (ASE) e lixiviação de solúveis em água e materiais não polimerizados.

\section{Conclusão}

Por meio da carga de polímero, espectroscopia ATR-IR e MEV, concluiu-se que a impregnação, seguida de polimerização in situ de MMA, usando GMA e MAA como aditivos, foi eficiente no preenchimento dos poros e capilares da madeira de guapuruvu. As alterações no pico de degradação térmica das fibras de madeira, a partir da inserção de GMA e MAA, indicaram reações químicas no recobrimento interno.

O compósito MMA apresentou incremento médio acima de $60 \%$ nas propriedades de higroscopicidade e estabilidade dimensional. A inserção dos aditivos mostrou efeitos positivos em todas as propriedades relacionadas a higroscopicidade, hidrofobicidade e estabilidade dimensional, com incrementos em torno de 67-90\%. Em geral o GMAé a melhor opção tanto para aumentar a hidrofobicidade e estabilidade dimensional, como para diminuir a higroscopicidade.

\section{Agradecimentos}

Os autores gostariam de agradecer ao $\mathrm{CNPq}$ (Conselho Nacional de Desenvolvimento Científico e Tecnológico), CAPES (Coordenação de Aperfeiçoamento de Pessoal de Nível Superior) e à Fundação Araucária, pelo apoio financeiro por intermédio de bolsas de pesquisa e de pós-graduação.

\section{Referências}

1. Correa, C. A., Fonseca, C. N. P., Neves, S., Razzino, C. A., \& Hage, J., Jr. (2003). Compósitos termoplásticos com madeira Polimeros, 13(3), 154-165. http://dx.doi.org/10.1590/S010414282003000300005 .

2. Ang, A. F., Zaidon, A., Bakar, E. S., Hamami, S. M., \& Anwar, U. M. K. (2009). Enhancing the properties of mahang (Macaranga spp.) wood through acrylic treatment in combination with crosslinker. Modern Applied Science, 3(11), 2-10. http:// dx.doi.org/10.5539/mas.v3n11p2.

3. Baysal, E., Yalinkilic, M. K., Altinok, M., Sonmez, A., Peker, H., \& Colak, M. (2007). Some physical, biological, mechanical, and fire properties of wood polymer composite (WPC) pretreated with boric acid and borax mixture. Construction \& Building Materials, 21(9), 1879-1885. http://dx.doi.org/10.1016/j. conbuildmat.2006.05.026.
4. Devi, R. R., \& Maji, T. K. (2012). Chemical modification of simul wood with styrene-acrylonitrile copolymer and organically modified nanoclay. Wood Science and Technology, 46(1-3), 299-315. http://dx.doi.org/10.1007/s00226-011-0406-2.

5. Devi, R. R., \& Maji, T. K. (2006). Effect of chemical modification with styrene and glycidyl methacrylate on the properties of pinewood. Indian Journal of Engineering and Materials Sciences, 13(2), 149-154. Recuperado em 26 de setembro de 2014, de http://nopr.niscair.res.in/handle/123456789/7228

6. Ding, W.-D., Koubaa, A., Chaala, A., Belem, T., \& Krause, C. (2008). Relationship between wood porosity, wood density and methyl methacrylate impregnation rate. Wood Material Science \& Engineering, 3(1-2), 62-70. http://dx.doi. org/10.1080/17480270802607947.

7. Ding, W.-D., Koubaa, A., \& Chaala, A. (2013). Mechanical properties of MMA-hardened hybrid poplar wood. Industrial Crops and Products, 46, 304-310. http://dx.doi.org/10.1016/j. indcrop.2013.02.004.

8. Islam, M. S., Hamdan, S., Hassan, A., Talib, Z. A., \& Sobuz, H.-J. (2014). The chemical modification of tropical wood polymer composites. Journal of Composite Materials, 48(7), 783-789. http://dx.doi.org/10.1177/0021998313477894.

9. Magalhães, W. L. E., \& Silva, R. R. (2004). Treatment of Caribbean pine by in situ polymerization of styrene and furfuryl alcohol. Journal of Applied Polymer Science, 91(3), 1763-1769. http://dx.doi.org/10.1002/app.13252.

10. Stolf, D. O., \& Lahr, F. A. R. (2004). Wood-polymer composite: physical and mechanical properties of some wood species impregnated with styrene and methyl methacrylate. Materials Research, 7(4), 611-617. http://dx.doi.org/10.1590/S151614392004000400015 .

11. Li, Y., Dong, X., Liu, Y., Li, J., \& Wang, F. (2011). Improvement of decay resistance of wood via combination treatment on wood cell wall: Swell-bonding with maleic anhydride and graft copolymerization with glycidyl methacrylate and methyl methacrylate. International Biodeterioration \& Biodegradation, 65(7), 1087-1094. http://dx.doi.org/10.1016/j.ibiod.2011.08.009.

12. Li, Y., Wu, Q., Li, J., Liu, Y., Wang, X.-M., \& Liu, Z. (2012). Improvement of dimensional stability of wood via combination treatment: swelling with maleic anhydride and grafting with glycidyl methacrylate and methyl methacrylate. Holzforschung, 66(1), 59-66. http://dx.doi.org/10.1515/HF.2011.123.

13. Carvalho, P. E. R. (2005). Guapuruvu: taxonomia e nomenclatura (Circular Técnica Embrapa Florestas, No. 104, pp. 1-10). Colombo: Embrapa. Recuperado em 26 de setembro de 2014, de http://ainfo.cnptia.embrapa.br/digital/bitstream/CNPF2009-09/43201/1/circ-tec104.pdf 
14. Zhang, H.-H., Cui, Y., \& Zhang, Z. (2013). Chemical treatment of wood fiber and its reinforced unsaturated polyester composites. Journal of Vinyl and Additive Technology, 19(1), 18-24. http:// dx.doi.org/10.1002/vnl.20321.

15. Devi, R. R., \& Maji, T. K. (2013). In situ polymerized wood polymer composite: effect of additives and nanoclay on the thermal, mechanical properties. Materials Research, 16(4), 954-963. http://dx.doi.org/10.1590/S1516-14392013005000071.

16. Hazarika, A., \& Maji, T. K. (2013). Effect of different crosslinkers on properties of melamine formaldehyde-furfuryl alcohol copolymer/montmorillonite impregnated softwood (Ficus hispida). Polymer Engineering and Science, 53(7), 1394-1404. http://dx.doi.org/10.1002/pen.23391.

17. Islam, M. S., Hamdan, S., Jusoh, I., Rahman, M. R., \& Talib, Z. A. (2011). Dimensional stability and dynamic young's modulus of tropical light hardwood chemically treated with methyl methacrylate in combination with hexamethylene diisocyanate cross-linker. Industrial \& Engineering Chemistry Research, 50(7), 3900-3906. http://dx.doi.org/10.1021/ie1021859.

18. Siau, J. F. (1984). Transport processes in wood. Berlin: Springer-Verlag.

19. Islam, M. S., Hamdan, S., Hasan, M., Ahmed, A. S., \& Rahman, M. R. (2012). Effect of coupling reactions on the mechanical and biological properties of tropical wood polymer composites (WPC). International Biodeterioration \& Biodegradation, 72(8), 108-113. http://dx.doi.org/10.1016/j.ibiod.2012.05.019.

20. Colom, X., \& Carrillo, F. (2005). Comparative study of wood samples of the Northern Area of Catalonia by FTIR. Journal of Wood Chemistry and Technology, 25(1-2), 1-11. http://dx.doi. org/10.1081/WCT-200058231.

21. Silverstein, R. M., Webster, F. X., \& Kiemle, D. (2005) Spectrometric identification of organic compounds. New York: John Wiley \& Sons.

22. Chen, Y., Fan, Y., Gao, J., \& Stark, N. M. (2012). The effect of heat treatment on the chemical and color change of black locust (Robinia pseudoacacia) wood flour. BioResouces, 7(1), 1157-1170.

23. Schwanninger, M., Rodrigues, J. C., Pereira, H., \& Hinterstoisser, B. (2004). Effects of short-time vibratory ball milling on the shape of FT-IR spectra of wood and cellulose. Vibrational Spectroscopy, 36(1), 23-40. http://dx.doi.org/10.1016/j. vibspec.2004.02.003.

24. Cademartori, P. H. G., Santos, P. S. B., Serrano, L., Labidi, J., \& Gatto, D. A. (2013). Effect of thermal treatment on physicochemical properties of Gympie messmate wood. Industrial Crops and Products, 45(2), 360-366. http://dx.doi. org/10.1016/j.indcrop.2012.12.048.

25. Calonego, F. W., Severo, E. T. D., \& Furtado, E. L. (2010). Decay resistance of thermally-modified Eucalyptus grandis wood at $140^{\circ} \mathrm{C}, 160^{\circ} \mathrm{C}, 180^{\circ} \mathrm{C}, 200^{\circ} \mathrm{C}$ and $220^{\circ} \mathrm{C}$. Bioresource
Technology, 101(23), 9391-9394. http://dx.doi.org/10.1016/j. biortech.2010.06.119. PMid:20655200.

26. Ferriol, M., Gentilhomme, A., Cochez, M., Oget, N., \& Mieloszynski, J. L. (2003). Thermal degradation of poly(methyl methacrylate) (PMMA): modelling of DTG and TG curves. Polymer Degradation \& Stability, 79(2), 271-281. http:// dx.doi.org/10.1016/S0141-3910(02)00291-4.

27. von Lampe, I., Schultze, D., \& Zygalsky, F. (2001). Thermal degradation of poly(methacrylic acid) and Y-Ba$\mathrm{Cu}$ polymethacrylate precursors for the preparation of high temperature superconductors. Polymer Degradation \& Stability, 73(1), 87-92. http://dx.doi.org/10.1016/S0141-3910(01)00072-6.

28. Zulfiqar, S., Zulfiqar, M., Nawaz, M., McNeill, I. C., \& Gorman, J. G. (1990). Thermal degradation of poly(glycidyl methacrylate). Polymer Degradation \& Stability, 30(2), 195203. http://dx.doi.org/10.1016/0141-3910(90)90075-I.

29. Vinu, R., \& Madras, G. (2008). Photocatalytic degradation of methyl methacrylate copolymers. Polymer Degradation \& Stability, 93(8), 1440-1449. http://dx.doi.org/10.1016/j. polymdegradstab.2008.05.018.

30. YongFeng, L., XiaoYing, D., ZeGuang, L., WanDa, J., \& YiXing, L. (2013). Effect of polymer in situ synthesized from methyl methacrylate and styrene on the morphology, thermal behavior, and durability of wood. Journal of Applied Polymer Science, 128(1), 13-20. http://dx.doi.org/10.1002/app.38099.

31. Devi, R. R., Maji, T. K., \& Banerjee, A. N. (2004). Studies on dimensional stability and thermal properties of rubber wood chemically modified with styrene and glycidyl methacrylate. Journal of Applied Polymer Science, 93(4), 1938-1945. http:// dx.doi.org/10.1002/app.20657.

32. Devi, R. R., \& Maji, T. K. (2007). Effect of glycidyl methacrylate on the physical properties of wood-polymer composites. Polymer Composites, 28(1), 1-5. http://dx.doi.org/10.1002/ pc.20265.

33. Ayrilmis, N., Benthien, J. T., \& Thoemen, H. (2012). Effects of formulation variables on surface properties of wood plastic composites. Composites. Part B, Engineering, 43(2), 325-331. http://dx.doi.org/10.1016/j.compositesb.2011.07.011.

34. Kaymakci, A., Ayrilmis, N., \& Gulec, T. (2013). Surface properties and hardness of polypropilene composites filled with sunflower stalk flour. BioResource, 8(1), 592-602. http:// dx.doi.org/10.15376/biores.8.1.592-602.

35. Mamiński, M., Król, M., McDonald, A., Mcllroy, D., Niraula, I., Czechowska, J., \& Parzuchowski, P. (2013). Thermally initiated solvent-free radical modification of beech (Fagus sylvatica) wood. Wood Science and Technology, 47(5), 10191031. http://dx.doi.org/10.1007/s00226-013-0555-6.

Enviado: Ago. 06, 2014 Revisado: Set. 26, 2014 Aceito: Dez. 18, 2014 